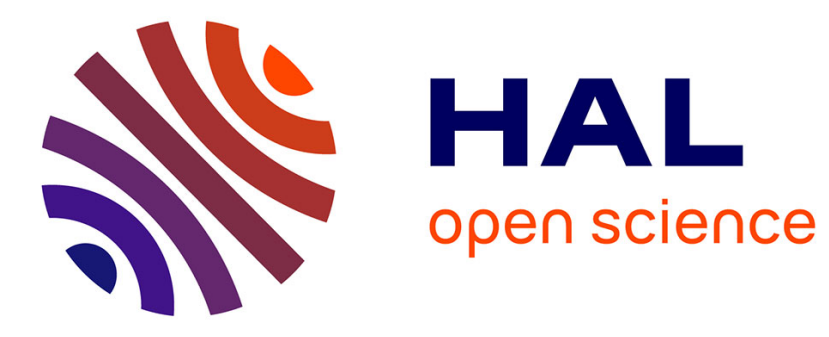

\title{
HMM Training Strategy for Incremental Speech Synthesis
}

\author{
Maël Pouget, Thomas Hueber, Gérard Bailly, Timo Baumann
}

\section{To cite this version:}

Maël Pouget, Thomas Hueber, Gérard Bailly, Timo Baumann. HMM Training Strategy for Incremental Speech Synthesis. Interspeech 2015 - 16th Annual Conference of the International Speech Communication Association, ISCA, Sep 2015, Dresden, Germany. pp.1201-1205. hal-01228889

\section{HAL Id: hal-01228889 \\ https://hal.science/hal-01228889}

Submitted on 14 Nov 2015

HAL is a multi-disciplinary open access archive for the deposit and dissemination of scientific research documents, whether they are published or not. The documents may come from teaching and research institutions in France or abroad, or from public or private research centers.
L'archive ouverte pluridisciplinaire HAL, est destinée au dépôt et à la diffusion de documents scientifiques de niveau recherche, publiés ou non, émanant des établissements d'enseignement et de recherche français ou étrangers, des laboratoires publics ou privés. 


\title{
HMM Training Strategy for Incremental Speech Synthesis
}

\author{
Maël Pouget ${ }^{1,2}$, Thomas Hueber ${ }^{1,2}$, Gérard Bailly ${ }^{1,2}$, Timo Baumann ${ }^{3}$ \\ ${ }^{1}$ CNRS/GIPSA-Lab, Grenoble, France \\ ${ }^{2}$ Univ. Grenoble Alpes/GIPSA-Lab, Grenoble, France \\ ${ }^{3}$ Universität Hamburg, Informatics Department, Natural Language Systems, Hamburg, Germany \\ 1,2 firstname.lastnamedgipsa-lab.fr, 3 baumann@informatik.uni-hamburg.de
}

\begin{abstract}
Incremental speech synthesis aims at delivering the synthetic voice while the sentence is still being typed. One of the main challenges is the online estimation of the target prosody from a partial knowledge of the sentence's syntactic structure. In the context of HMM-based speech synthesis, this typically results in missing segmental and suprasegmental features, which describe the linguistic context of each phoneme. This study describes a voice training procedure which integrates explicitly a potential uncertainty on some contextual features. The proposed technique is compared to a baseline approach (previously published), which consists in substituting a missing contextual feature by a default value calculated on the training set. Both techniques were implemented in a HMM-based TextTo-Speech system for French, and compared using objective and perceptual measurements. Experimental results show that the proposed strategy outperforms the baseline technique for this language.
\end{abstract}

Index Terms: HMM-based speech synthesis, incremental, TTS, HTS, prosody

\section{Introduction}

Incremental Text-To-Speech (iTTS) systems aim at starting delivery of the synthetic voice before the full sentence context becomes available, e.g. while a user is still typing the text to vocalize. Contrary to a conventional TTS, the synthesis follows the text input, words after words (potentially with a delay of one word). This 'synthesis-while-typing' approach is illustrated in Figure 1. By reducing the latency between text input and speech output, iTTS should enhance the interactivity of communication. In particular, it should improve the user experience of people with communication disorders who use a TTS system in their daily life, as a substitute voice. Besides, iTTS could be chained with incremental speech recognition systems, in order to design highly responsive speech-to-speech conversion systems (for application in automatic translation, silent speech interface, real-time enhancement of pathological voice, etc.).

To our best knowledge, the concept of incremental speech synthesis was initially formulated in [1] in the context of dialogue systems. However, in the proposed proof-of-concept, the speech generation was delivered incrementally but was generated in a non-incremental way. In [2], Baumann \& Schlangen proposed the first complete software architecture dedicated to incremental speech processing (including recognition, dialogue management and TTS modules). Another proof-of-concept based on the reactive HMM-based parameter generation system MAGE [3] was described in [4].

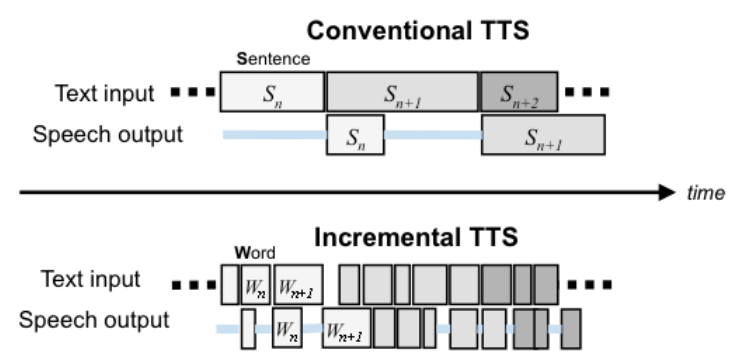

Figure 1: Conventional versus incremental TTS

One of the main remaining challenges of iTTS systems is the online estimation of the target prosody from an incomplete sentence (and therefore an uncertain - incrementally unveiled syntactic structure). In conventional TTS, target prosody is typically calculated from long-range contextual features [5], [6], extracted from the text by morphological and syntactic analyzers. Considering a current segment as reference (typically a phoneme), some of these features refer to its left context (i.e. the 'past'); some of them refer to its right context (i.e. the 'future'). These features can, for instance, be the part-of-speech tag (POS) of the next word, or the number of remaining words before the end of the current sentence. Indeed, such features related to the right context are usually not available in incremental processing. Therefore, strategies should be developed to deal with these 'missing' features and predict acceptable prosody from an 'incomplete' sentence. This is the general scope of the present study.

In [7], [8], Baumann first evaluated the impact of potentially missing features on the quality of the estimated prosody, in the context of HMM-based speech synthesis, for English and German languages. Then, the author proposed a strategy for predicting a 'default' value for these missing features (this strategy is therefore referred to as the 'Default' strategy here). This strategy exploits the decision trees that are classically used in HMM-based speech synthesis in the state clustering procedure. The goal of this present study is twofold. First, we evaluate this strategy [7] (briefly recalled in Section 2) for French language, which has different prosodic characteristics than English and German (for instance, French can be considered to have no lexical stress[9]). Second, we propose another approach for dealing with missing contextual features, also in the context of HMM-based speech synthesis (Section 3). Contrary to [7], our approach does not aim at recovering the missing features at synthesis time. It rather consists in integrating a potential uncertainty on some features when building the synthetic voice (i.e. when training the HMM set). This strategy is here referred to as the 'Joker' strategy. The two strategies were implemented in an HMM-based TTS for French 
language, and compared using objective measurements and a perceptual listening test (Section 4).

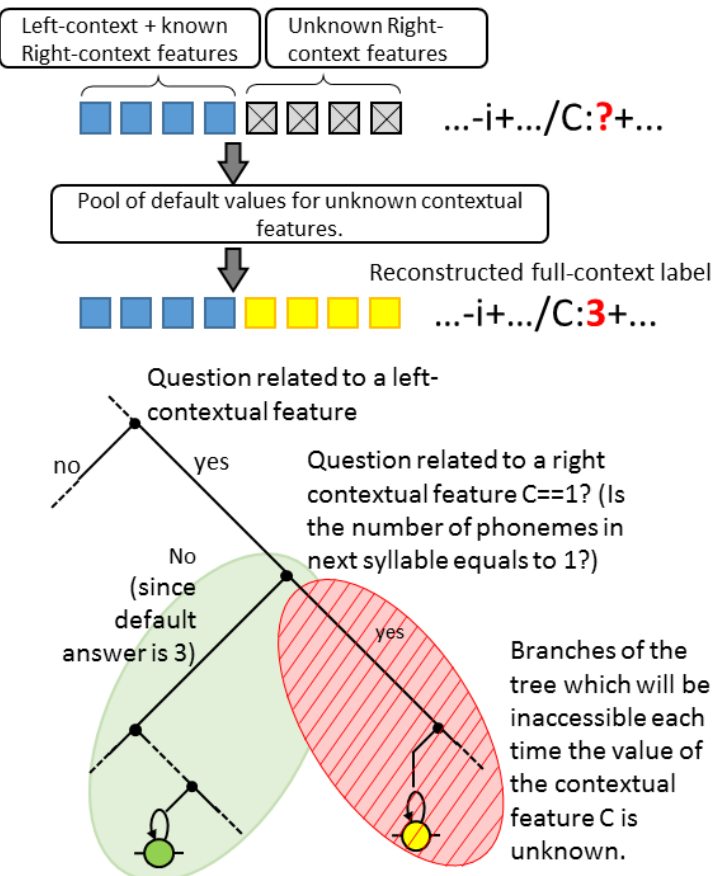

Figure 2. Procedure for recovering full context labels from incomplete labels and clustering tree exploration when building unseen labels with the default strategy.

\section{Baseline strategy: 'Default'}

In most implementations of HMM-based TTS (such as [10] or [11]), each speech unit is a phone in context. The context is described by a set of segmental features such as the identity of the current and adjacent phonemes, and suprasegmental features, such as the POS of the current and adjacent words, the position of the word in the current breath group, etc. Since it is very difficult to build a training dataset covering all possible contexts, clustering techniques are used to group some HMM states and share their model parameters (similarly to ASR systems). The most widely used technique is tree-based clustering [12]. Each node of the tree is associated with a context-related question, such as 'R-SYLL-NB-PHON==3' ("Are there 3 phonemes in the next syllable?"). The pertinence of the context-related questions and the structure of the tree are learnt automatically from the training dataset with respect to a specific criterion, such as the Minimum Description Length [13]. At synthesis time, the decision tree is extensively used when building the so-called 'unseen models' (i.e. corresponding to contexts with no acoustic observations in the training set).

In [7], Baumann proposed to exploit these decision trees to recover the missing contextual features at synthesis time. The procedure, which is illustrated in Figure 2, can be summarized as follows. First, a set of full-context HMMs (i.e. HMMs modeling each phoneme with information about its left and right contexts) is trained using a standard procedure (including tree-based clustering). Then, a 'default answer' is assigned to each contextual feature related to the right context (which might be unknown in incremental processing). This 'default answer' is calculated from the training set, by averaging the answers observed at each node of the decision tree associated with a question on the right context. For numerical features (e.g. the number of words before the end of the sentence), the default answer is the mean value calculated across the training dataset; for symbolic features (e.g. the POS tag of the next word), the default answer is the most common value observed in the training dataset.

This strategy gives encouraging results and works with preexisting voices that were not trained with the incremental usecase in mind, but presents a major disadvantage. By imposing a default value to some contextual features, some branches of the clustering trees become totally unexplored when building the 'unseen models' (as shown by the red dashed zone in Figure 2). Therefore, only a limited number of HMM states are used at synthesis time. In other words, the fine-grained modeling of the training dataset based on a rich set of contextual features is not exploited here. In the next section, we present another strategy to deal with missing contextual features in the context of HMMbased incremental synthesis.

\section{Proposed strategy: 'Joker'}

In the proposed approach, the potential uncertainty on rightcontextual features is handled during voice training rather than during the synthesis process, as in the 'Default' strategy. The proposed technique aims at considering a contextual feature that could potentially be missing as 'relevant' information that can be explicitly used when describing the linguistic context. Besides, when clustering the pool of HMM-states, we evaluate the need of tying model parameters among all contexts potentially sharing the same missing features. This training procedure results in a set of context-dependent HMMs that are likely to be slightly less accurate than full-context models (for instance, they are expected to deliver a neutral intonation for situations where the right context would trigger very different patterns). However, the 'Joker' strategy may lead to better perceptual results since there is no risk it uses an incorrect fullcontext model, as in the 'Default' strategy.

The 'Joker' has been implemented in the HTS framework as follows. First, the training corpus is labeled by introducing a so-called 'Joker' value (specified by the \# character) to each contextual feature which cannot be determined when processing the text incrementally. This notably affects all the contextual features requiring information about the next word. As an example, let us consider the label associated with the phoneme in the last syllable of the current word, and the right-contextual feature 'number of phonemes in the next syllable (usually denoted by the symbol ' $C$ ' in HTS). Since the value of this feature is unknown when processing the text incrementally, the 'Joker' tag is inserted in the label such as: “...-p+.../C:\#..." (other contextual features are omitted for clarity). Then, a set of context-dependent HMMs is trained using a standard procedure (similarly to a non-incremental system). The Joker tag (\#) is simply considered as a possible value for some contextual features.

A tree-based clustering procedure is then applied to deal with data sparsity. However, contrary to a non-incremental system, we introduce questions about the possible known/unknown characteristic of each contextual feature. In the HTS format, this can be written as: " $Q S$ " $R$ nb phone in next syllabe is unknown" $\{* / C: \#\}$ " where $C$ stands for the number of phonemes in the next syllable. At the end of the clustering procedure, the parameters of some models/states sharing a common missing feature are expected to be tied together. The rest of the training procedure, as well as the synthesis procedure are similar to a non-incremental system. 
As shown in Figure 3, one of the main advantages of the 'Joker' strategy compared to the 'Default' strategy, is a better utilization of the decision tree when building the unseen models. Even if a question related to a missing contextual feature in used at a specific node of the tree, the label can continue to 'go down' to all sub-branches of this node. Therefore, in this case, all HMM states are reachable. This should result in more variety in the estimated trajectories, compared to the 'Default' strategy, as shown by the experimental results described in the next sections.

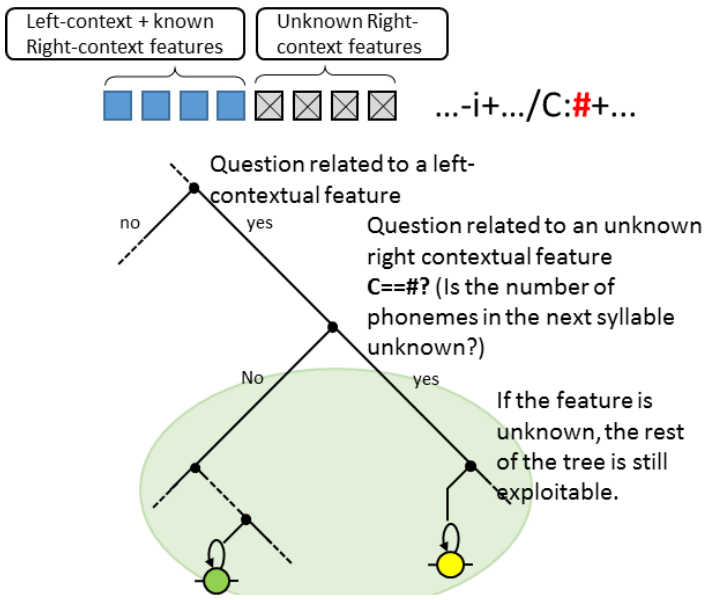

Figure 3. Usage of the decision tree for building unseen models using the 'Joker' strategy.

\section{Experimental protocol and results}

\subsection{HMM-based TTS system for French language}

The two strategies described in the previous sections were evaluated in the context of an HMM-based TTS system, developed in our group for French language. The specificities of this system are the following. The audio material used for training was extracted from an audiobook of the novel "Le tour du Monde en 80 jours" by Jules Verne (this corpus was also used in [14]). This corpus contains $3 \mathrm{~h} 17 \mathrm{mn}$ of speech data, after silence being removed. Phonetization as well as morphological and syntactic analyses of the text transcriptions were achieved using the linguistic front-end COMPOST [15]. The contextual features considered in this study are listed below (the features which are potentially missing when only the past and current words are known are written in bold):

- Identity of the $\mathrm{n}-2, \mathrm{n}-1, \mathrm{n}$ (current), $\mathbf{n}+\mathbf{1}, \mathbf{n}+\mathbf{2}$ phoneme

- Position of current phoneme in the current syllable (forward \& backward)

- Number of phonemes in the previous/current/next syllable.

- Identity of the vowel of the current syllable

- Position of the current syllable in the word (forward \& backward)

- Position of the current syllable in the sentence (forward \& backward)

- POS-tag of previous/current/next word (always missing)

- Number of syllables in the previous/current/next word (always missing)

- Position of the current word in the sentence (forward \& backward)

- Sentence type (assertion, wh-question, full question, etc.)
An initial segmentation of the audio recordings at phonetic level was obtained using a forced-alignment procedure and was then post-processed manually. In our system, the full-band spectral envelope is parameterized using a "Harmonic plus Noise Model" (HNM) [16] (and not mel-cepstral coefficients as in [17]), following the implementation detailed in [18] (p.82). Each acoustic observation (extracted each $5 \mathrm{~ms}$ ) is a 93dimensional vector composed of the fundamental frequency $f_{0}$, a $\left(12^{\text {th }}\right.$-order $)$ LSF-modeling of the harmonic component of the spectral envelope (defined for voiced frames only), and a ( $16^{\text {th }}$ order) LSF-modeling of the residual spectrum, completed by first and second derivatives. A set of context-dependent HMMs (5 emitting states for the acoustic models) were trained on this corpus using the HTS toolkit [10] and a standard procedure. Global variance optimization was not used in this study.

Similarly to [7], we calculated the percentage of use of each right-contextual questions for clustering the training set, for spectrum-related streams, pitch and duration. As shown in Figure 4, we observed approximately the same pattern for French and German (see Figure 2 in [7]). As in [7], most of the questions recruited for clustering the spectrum-related parameters were related to the quinphone context. However, for pitch and duration, more questions related to current and next word were used for French than for German.

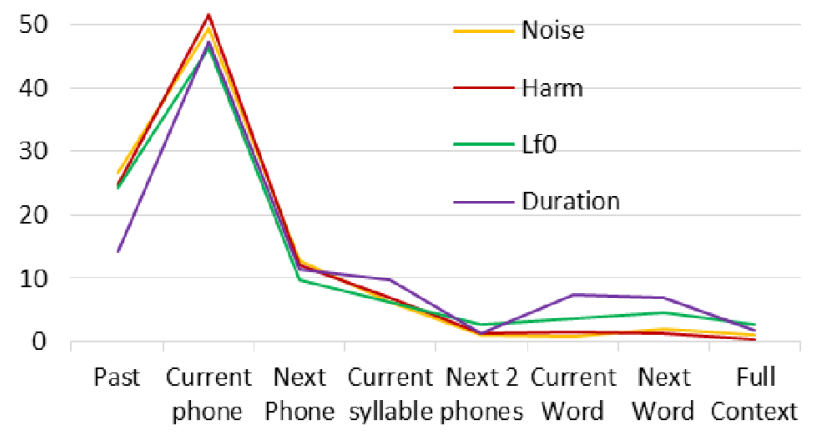

Figure 4. Percentage of use of right-contextual questions used in the decision tree for clustering the training set, for spectrum (Harmonic + Noise), pitch and duration

\subsection{Objective evaluation}

The two strategies considered in this study were first evaluated using as set of objective measurements. A subset of 165 test sentences was randomly selected from the corpus (and removed from the training set). These sentences were first synthesized using a non-incremental approach. The resulting acoustic feature vectors (i.e. spectrum, $f_{0}$ and duration) were considered as the 'best possible result'. In other words, we assume that incremental processing will systematically lead to lower performance than non-incremental processing (a similar assumption was made in [7]). The 165 test sentences were then synthesized using both 'Default', and 'Joker' strategies. The accuracy of the estimated spectrum was evaluated by calculating a mel-cepstral distortion [19] in $\mathrm{dB}$ defined such as:

$$
\operatorname{MCD}\left(\mathbf{y}_{t}^{S}, \boldsymbol{y}_{t}^{N I}\right)=\frac{1}{T} \frac{10}{\ln (10)} \sum_{t=1}^{t=T} \sqrt{2 \sum_{d=0}^{D}\left(\mathbf{y}_{t}^{S}-\boldsymbol{y}_{t}^{N I}\right)^{2}}
$$

where $\mathbf{y}_{t}^{S}$ and $\mathbf{y}_{t}^{N I}$ are respectively vectors of $D+1$ mel-ceptral coefficients estimated using the $S$ incremental strategy and the baseline non-incremental $(N I)$ approach and $T$ the number of frames in the utterance. These coefficients were derived from the HNM-model of the spectrum (section 4.1) using the SPTK 
toolkit [20]. A perceptual-based measure [21] of the difference (in cents) (also used in [22]) between incremental and nonincremental approaches in terms of $f_{0}$ was calculated for each utterance such as:

$$
E_{f_{0}}=\frac{1}{T} \sum_{t=1}^{T} 1200 \log _{2}\left|f_{0}^{S}(t) / f_{0}^{N I}(t)\right|
$$

The timing distortion induced by non-incremental processing was evaluated by calculating for each test sentence the log duration ratio [23] such as:

$$
E_{d u r}=\frac{1}{P} \sum_{p=1}^{P} \log \left(\mathrm{d}_{\mathrm{NI}_{\mathrm{p}}} / \mathrm{d}_{\mathrm{S}_{\mathrm{p}}}\right)
$$

where $\mathrm{d}_{\mathrm{NI}_{p}}$ and $\mathrm{d}_{\mathrm{S}_{p}}$ are the estimated phoneme duration obtained using non-incremental and incremental approaches, respectively, and $P$ is the number of phonemes in the utterance. For each metric $\left(M C D, E_{f 0}, E_{d u r}\right)$, the statistical significance of the difference between 'Default' and 'Joker' strategies was assessed using a paired t-test. Experimental results are presented in Table 1.

Table 1: Objective differences between 'Default' and 'Joker' for spectrum, $f 0$ and phone duration, averaged across the test set ( \pm standard deviation).

\begin{tabular}{|l|c|c|c|}
\hline & Default & Joker & $\begin{array}{c}\text { Joker vs. } \\
\text { Default }\end{array}$ \\
\hline MCD (dB) & $0.78 \pm 0.26$ & $0.94 \pm 0.15$ & $* * *$ \\
\hline $\mathrm{E}_{f 0}$ (cents) & $197.4 \pm 88.7$ & $178.2 \pm 78.4$ & NS \\
\hline$E_{\text {dur }}$ & $0.20 \pm 0.06$ & $0.17 \pm 0.04$ & $* * *$ \\
\hline
\end{tabular}

In terms of spectrum estimation, the distortions observed with both incremental strategies are relatively small (less than 1 $\mathrm{dB})$. This result is compatible with the study of [24] showing that a look ahead of two phonemes (i.e quinphone modeling with no long-range contextual features) is enough to accurately estimate the target spectrum. The highest distortion was obtained with the 'Joker' strategy (which can therefore be considered as slightly less accurate than the default strategy). However, the difference between the two strategies, even statistically significant, is tiny $(0.16 \mathrm{~dB})$. An opposite effect was observed for pitch and segment duration (which are more closely related to prosody). Also, and despite statistical significance, the differences between the two strategies remain too small to conclude to a perceptual difference (i.e. with less than 20 cents for $f 0$ ). Therefore, a listening test was conducted to study in more detail potential perceptual differences between the two strategies.

\subsection{Perceptual evaluation}

The perceptual evaluation was conducted with a ranking listening test, similar to [25] and [26]. For each trial, the subject was asked to sort 3 sound samples, according to its "naturalness". These sound samples correspond to the same sentence synthesized respectively with the non-incremental approach, the 'Default' approach, and the proposed 'Joker' approach. Two stimuli used in this test are submitted as supplementary material (exampleX_S.wav with $X=\{1,2\}$, $S=\{$ joker, default, nonIncremental $\}$ ). For each test sentence, the user interface used for this test was composed of a ranking $\mathrm{X} / \mathrm{Y}$ area, in which each listener was asked to 'drag and drop' the 3 audio samples to rank. Each sample was represented by a 'push button' allowing to listen to it, as many times as required. The $\mathrm{X}$-axis of the ranking area was a continuous scale (ranging from 0 to 5). A set of 5 labels ("very bad, bad, middle, good, very good") was nevertheless added to help the subject in the ranking process (the scale can thus be considered as semi-continuous). The position on the $\mathrm{Y}$-axis was not taken into account (as told to the subject). The test was conducted in a quiet room with the same headphones, with 18 native speakers of French, with no particular expertise in speech synthesis. They were asked to evaluate a set of 12 sentences (resulting in 36 stimuli in total). These sentences were randomly extracted from the test set (the shortest selected sentence was 14 syllables long, the longest was 27 syllables long). For each trial and each participant, the presentation order of the stimuli was randomized. Both parametric (ANOVA) and non-parametric (Kruskal-Wallis) tests were conducted to assess the statistical significance of the results. These tests considered the X-position on the ranking area as the continuous variable to explain, the 3-level explicative variable Strategy (with the possible values 'nonincremental', 'default', 'joker'), and a random Listener effect on the intercept. Since the main effect of the factor Strategy was significant $(\mathrm{p}<0.005)$, post-hoc analyses were conducted to test the contrast between 'Default' and 'Joker' strategies. Results are presented in Figure 5.

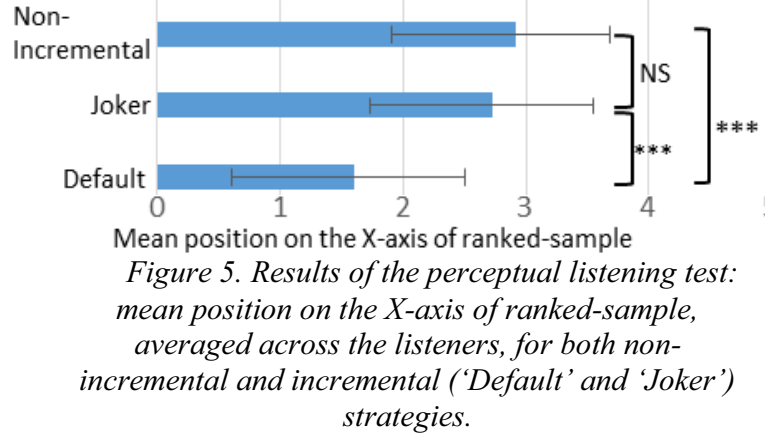

As expected, the best-ranked samples were those obtained with the non-incremental approach (i.e. with a complete set of contextual features). The proposed 'Joker' strategy outperforms significantly the baseline ('default') strategy ( 2.8 vs. 1.5 , $\mathrm{p}<0.005$ ). This supports the benefit of considering explicitly the uncertainties about right context when building the synthetic voice. Interestingly, no statistically significant difference was observed between the non-incremental and the 'Joker' strategy. Amongst other possible causes, this could be explained by a 'ceiling effect', due to the intrinsic quality of the baseline HMM-based synthesis (with a mean score of 3 ).

\section{Conclusion and perspectives}

This study describes a strategy for dealing with missing contextual features, for incremental HMM-based speech synthesis. This strategy consists in integrating a potential uncertainty on some contextual features when training the HMM set. This approach was compared to the baseline technique proposed in [7]. Both strategies were implemented in an HMM-based TTS system for French. A perceptual test shows that the proposed strategy outperforms the baseline technique for that language. Future work will focus on the evaluation of the proposed strategy for other languages, such as English and German (which were considered in [7]). In order to build a complete incremental TTS system, we will also combine the proposed technique with and 'incremental text parsing' front-end. Such module could be inspired by some approaches developed for incremental text processing and syntactic parsing [27]. 


\section{References}

[1] J. Edlund, "Incremental speech synthesis," in Proceedings of Swedish Language Technology Conference, Stockholm, Sweden, 2008, pp. 53-54.

[2] T. Baumann and D. Schlangen, "The INPROTK 2012 release," in Proceedings of NAACL-HLT Workshop on Future Directions and Needs in the Spoken Dialog Community: Tools and Data, Stroudsburg, PA, USA, 2012, pp. 29-32.

[3] M. Astrinaki, N. d' Alessandro, and T. Dutoit, "MAGE: A Platform for Performative Speech Synthesis New Approach in Exploring Applications Beyond Text-ToSpeech," in Proceedings of The Listening Talker Workshop, Edinburgh, Scotland, 2012, p. 53.

[4] Y. Rybarczyk, T. Cardoso, J. Rosas, L. CamarinhaMatos, N. d' Alessandro, J. Tilmanne, M. Astrinaki, T. Hueber, R. Dall, T. Ravet, A. Moinet, H. Cakmak, O. Babacan, A. Barbulescu, V. Parfait, V. Huguenin, E. Kalayc1, and Q. Hu, "Reactive Statistical Mapping: Towards the Sketching of Performative Control with Data," in Innovative and Creative Developments in Multimodal Interaction Systems, vol. 425, Springer Berlin Heidelberg, 2014, pp. 20-49.

[5] D. Büring, "Syntax, information structure and prosody," in The Cambridge Handbook of Generative Syntax, Marcel den Dikken, 2013, pp. 860-895.

[6] C.-Y. Tsai, C.-K. Kuo, Y.-R. Wang, S.-H. Chen, I.-B. Liao, and C.-Y. Chiang, "Hierarchical prosody modeling of English speech and its application to TTS," in Proceedings of Co-ordination and Standardization of Speech Databases and Assessment Techniques (COCOSDA), 2014 17th Oriental Chapter of the International Committee for the, Pukhet, Thaïlande, 2014, pp. 1-6.

[7] T. Baumann, "Decision tree usage for incremental parametric speech synthesis," in Proceedings of ICASSP, Florence, Italy, 2014, pp. 3819-3823.

[8] T. Baumann, "Partial Representations Improve the Prosody of Incremental Speech Synthesis," in Proceedings of Interspeech, Singapore, 2014, pp. 2932 2936.

[9] M. Rossi, Le Français, langue sans accent?, Studia Phonetica Montréal., vol. 15. 1980.

[10] K. Tokuda, T. Yoshimura, T. Masuko, T. Kobayashi, and T. Kitamura, "Speech parameter generation algorithms for HMM-based speech synthesis," in Proceedings of ICASSP, Istanbul, Turkey, 2000, vol. 3, pp. $1315-1318$.

[11] T. Yoshimura, K. Tokuda, T. Masuko, T. Kobayashi, and T. Kitamura, "Simultaneous modeling of spectrum, pitch and duration in HMM-based speech synthesis," in Proceedings of Eurospeech, Budapest, Hungary, 1999, pp. 2347-2350.

[12] S. J. Young, J. J. Odell, and P. C. Woodland, "Treebased State Tying for High Accuracy Acoustic Modelling," in Proceedings of the Workshop on Human Language Technology, Stroudsburg, PA, USA, 1994, pp. 307-312.

[13] K. Shinoda and T. Watanabe, "MDL-based contextdependent subword modeling for speech recognition," $J$. Acoust. Soc. Jpn., vol. 21, no. 2, pp. 79-86, 2000.

[14] G. Bailly and C. Gouvernayre, "Pauses and respiratory markers of the structure of book reading," in
Proceedings of Interspeech, Portland, OR, USA, 2012, pp. 2218-2221.

[15] M. Alissali and G. Bailly, "COMPOST: a client-server model for applications using text-to-speech systems.," in Proceedings of European Conference on Speech Communication and Technology, Berlin, Germany, 1993, pp. 2095-2098.

[16] Y. Stylianou, "Harmonic plus Noise Models for Speech combined with Statistical Methods, for Speech and Speaker Modification," Ph.D. thesis, Ecole Nationale superieure des télécommunications, Paris, France, 1996.

[17] "The HTS toolkit." [Online]. Available: http://hts.sp.nitech.ac.jp/.

[18] T. Hueber, "Reconstitution de la parole par imagerie ultrasonore et vidéo de l'appareil vocal: vers une communication parlée silencieuse," Ph.D. thesis, Université Pierre et Marie Curie, Paris, France, 2009.

[19] R. F. Kubichek, "Mel-cepstral distance measure for objective speech quality assessment," in Proceedings of Communications, Computers and Signal Processing, IEEE Pacific Rim Conference on, Victoria, British Columbia, Canada, 1993, vol. 1, pp. 125-128 vol.1.

[20] T. Fukada, K. Tokuda, T. Kobayashi, and S. Imai, "An adaptive algorithm for mel-cepstral analysis of speech," in Proceedings of ICASSP, San Francisco, CA, USA, 1992, vol. 1, pp. 137-140 vol.1.

[21] I. Peretz and K. L. Hyde, "What is specific to music processing? Insights from congenital amusia," Trends Cogn. Sci., vol. 7, no. 8, pp. 362-367, 2003.

[22] M. Astrinaki, N. d' Alessandro, B. Picart, T. Drugman, and T. Dutoit, "Reactive and continuous control of HMM-based speech synthesis," in Proceedings of Spoken Language Technology Workshop (SLT), IEEE, Miami, FL, USA, 2012, pp. 252-257.

[23] N. W. Campbell, "Segment durations in a syllable frame," J. Phon., vol. 19, no. 1, pp. 37-47, 1991.

[24] S. Le Maguer, "Evaluation experimentale d'un systeme statistique de de la parole, HTS, pour la langue francaise.," Ph.D. thesis, Université de Rennes 1, Rennes, France, 2013.

[25] G. Bailly and I. Gorisch, "Generating German intonation with a trainable prosodic model," in Proceedings of Interspeech, Pittsburgh, PA, USA, 2006, pp. 2366-2369.

[26] H. R. Pfitzinger, "Local Speech Rate As A Combination Of Syllable And Phone Rate," in Proceedings of International Conference on Spoken Language Processing (ICSLP), Sydney, Australia, 1998, pp. $1087-$ 1090.

[27] N. Beuck, A. Köhn, and W. Menzel, "Predictive incremental parsing and its evaluation," in Computational Dependency Theory, vol. 258, Kim Gerdes, Eva Hajičová, Leo Wanner, 2013, p. 186. 